\title{
Regulation of autophagy by miRNAs in human diseases
}

\author{
Sounak Ghosh Roy ${ }^{1}$ (D)
}

Received: 22 July 2021 / Accepted: 6 October 2021 / Published online: 16 October 2021

(c) Archana Sharma Foundation of Calcutta 2021

\begin{abstract}
Autophagy is a homeostatic process designed to eliminate dysfunctional and aging organelles and misfolded proteins through a well-concerted pathway, starting with forming a double-membrane vesicle and culminating in the lysosomal degradation of the cargo enclosed inside the mature vesicle. As a vital sentry of cellular health, autophagy is regulated in every human disease condition and is an essential target for non-coding RNAs like microRNAs (miRNAs). miRNAs are short oligonucleotides that specifically bind to the 3'-untranslated region (UTR) of target mRNAs, thus leading to mRNA silencing, degradation, or translation blockage. This review summarizes the recent findings regarding the regulation of autophagy and autophagy-related genes by different miRNAs in various pathological conditions, including cancer, kidney and liver disorders, neurodegeneration, cardiovascular disorders, infectious diseases, aging-related conditions, and inflammation-related diseases. As miRNAs are being identified as prime regulators of autophagy in human disease, pharmacological molecules and traditional medicines targeting these miRNAs are also being tested in disease models, thus initiating a new series of therapeutic interventions targeting autophagy.
\end{abstract}

Keywords Autophagy $\cdot$ miRNA $\cdot$ autophagomIRs $\cdot$ Non-coding RNAs

\section{Introduction-autophagy and miRNAs}

Macroautophagy is a homeostatic recycling process where damaged and aged organelles and macromolecules are enclosed in double-membrane vesicles (DMVs) and degraded through a lysosomal process. Despite other variations like microautophagy and chaperone-mediated autophagy (CMA) being prevalent in cellular housekeeping-macroautophagy is the most common and is interchangeably used with 'autophagy.' Autophagy comprises distinct stages, starting with induction (ULK complex), and followed in order by vesicle nucleation (BECLIN-1, UVRAG, RAB5A), elongation (LC3B, ATGs4, 7, 5, 12, 16, 16L1, 10A), retrieval (ATGs2B, 9A), lysosomal fusion (LAMP2A), and finally degradation of the autophagic 'cargo' (SQSTM1/P62) [7]. While it is commonly initiated during stress conditions (infection, radiation, hypoxia), it is also

Corresponding Editor: Somnath Paul; Reviewers: Oishee Chakrabarti, Miao-Der Chen.

Sounak Ghosh Roy

sounak.ghoshroy@yale.edu

1 Department of Internal Medicine - Nephrology, Yale School of Medicine, New Haven, CT, USA well established that regulation of autophagy is a crucial checkpoint in cancer, viral infection [20], lung disease [61], innate immunity [55], kidney disease [6], diabetes, obesity, osteoarthritis [48], inflammation-related disorders [18], cardiovascular disease [1], aging-related disease, lysosomal storage disorders, and neurodegeneration [63, 64, 99].

Alongside small nucleolar RNAs (snoRNAs), Piwi-interacting RNAs (piRNAs), long non-coding RNAs (lncRNAs), circular RNAs (circRNAs), microRNAs (miRNAs) are essential non-coding RNAs estimated to regulate $\sim 30 \%$ of the human genome in a post-transcriptional manner. These small oligonucleotides (18-25 nt long), usually found in the intergenic regions or the introns, bind to specific areas (miRNA responsive element/ MRE) primarily within the 3'UTR of a target mRNA and results in mRNA silencing. This miRNA-induced mRNA silencing can follow different mechanisms (RISC-induced mRNA cleavage, mRNA sequestration in $\mathrm{P}$ bodies, blockage of translation) depending upon the complementarity between the miRNA 'seed' (6-8nt) sequence and the mRNA target sequence. miRNAs can frequently downregulate multiple target mRNAs, enabling the regulation of diverse cellular pathways like oxidative stress, cell death, and differentiation [5, 24]. Almost $100 \mathrm{miR}$ NAs have been identified that regulate different steps of the 
autophagy pathway, i.e., Induction, Nucleation, Elongation, Retrieval, Fusion. Interestingly, some of these genes act as targets for multiple miRNAs, also referred to as autophagomIRs [2, 4, 25]. For instance, the nucleation genes Beclin-1 (miR-30a, 376b, 519a), Uvrag (miR-630, 374a); elongation genes Atg4 (miR-101, 376b), Atg5/12 (miR-30a, 630, 374a, 181a); fusion gene Lamp2/Rab7 (miR-98, 102, 124, 130, 142); and finally, the lysosomal marker Sqstml (miR-17, $20,93,106)$ are all regulated by different autophagoMIRs. It is also noteworthy that some miRNAs (30a, 376b, 630a) can downregulate multiple genes in the autophagy pathway, thus increasing the extent to which these short oligonucleotides can suppress autophagy. Since autophagy is regulated in various pathological conditions ranging from cancer to microbial infections and kidney disorders, miRNAs regulating autophagy hold important cues for treating these diseases [21,22]. This minireview is dedicated to summarizing the most recent findings on the regulation of autophagomIRs in different diseases and the utilization of small molecules and traditional medicines in harnessing these miRNAs as targets for potential therapies.

\section{Ageing}

A few studies point to the critical role that miRNAs can potentially execute in the progression of aging. In Caenorhabditis elegans, miR-83/ miR-29 is activated by a transcription factor HSF-1 in the intestine and body wall muscles. This event blocks autophagy-miR-83 inhibits autophagy-related CUP5 protein - and facilitates aging in these tissues. Interestingly, miR- 83 is transported to other tissues through the coelomic fluid in C. elegans [103]. Furthermore, miRNAs regulating autophagy have been implicated in other aging-related processes like intervertebral disc degeneration (IDD) in humans [36]. In most of these studies, upregulation of miRNAs resulting in low autophagy levels has been linked to IDD's possible causes. For instance, in some patient samples, miR-21 blocks PTEN-mediated autophagy and promotes extracellular matrix (ECM) degradation, thus facilitating IDD progression; in few other cases, miR-21 increases the interleukin (IL)-6 inflammatory response to blunt autophagy [43, 80]. Similarly, miRNAs (miR-129-5p, miR-185) blocking other upstream regulators of autophagy, like BECN-1 and GALECTIN-3, are also deemed responsible for IDD in other reports [95, 101]. However, proteins directly involved in the autophagy pathway are also regulated by miRNAs in human IDD. In different scenarios, autophagy-related genes (ATGs) 5 and 7 are targeted by non-coding RNAs like miR-153-3p, miR202-5p, and miR-210 in human IDD samples [12, 76, 81]. miRNAs regulate cardiac autophagy in 19-week-old healthy rats treated with an autophagy activator Rapamycin, the first
FDA-approved longevity drug [59]. This data highlights the importance of miRNAs in regulating cardiac autophagy during the treatment of aging with anti-senescence therapies.

\section{Cancers}

Autophagy is a vital homeostasis pathway and is deregulated in various cancers. One of the early reports investigating the role of autophagomIRs in cancer has linked miR-30a to the downregulation of autophagy in the human breast cancer cell lines (MDA-MB-468), lung cancer cell lines (T98G), and glioma cell lines (H1299). miR-30a inhibits the function of an autophagy gene Beclin1; induction of miR-30a activity using mimics has been shown to blunt even Rapamycininduced autophagy in these cell lines [104]. The complicated nature of the interplay between miRNA and autophagy in cancer cells lies in many reports that show miRNAs inducing pro-tumor autophagy pathways. miR-638 inhibits a vital regulator of the $\mathrm{Wnt} / \beta$-catenin signaling, DACT3, a negative regulator of autophagy, in esophageal squamous cancer (KYSE450) and breast cancer (MCF-7) cell lines [62]. In breast cancer cells (MCF-7, MDA-MB-231), miR-224-5p inhibits the autophagy pathway by blocking SMAD4 activity - thus identifying the miR-224-5p/SMAD4 pathways as a potential target for therapy [15].

Low miR-23b activity, and upregulation of its specific target ATG12, have been recorded in radioresistant pancreatic cancer cells. Indeed, the administration of miR-23b mimics has blocked autophagy and sensitized pancreatic cancer cells to radiotherapy, thus highlighting the role of miRNAs in anticancer therapy [78]. Interestingly, miRNAs' part is not always antagonistic to the autophagy pathway as far as pancreatic cancer or even cancer therapy is concerned. miR221 blocks histone deacetylase (HDAC) activity to induce autophagy and apoptosis in hamster and human pancreatic cancer cell lines. Thus, miR-221 could be helpful to attenuate proliferation in pancreatic cancer models [92]. Ironically, HDAC inhibitors (HDACi) which are otherwise used to treat many solid tumors, can also provoke chemoresistance by upregulation of autophagy. Remarkably, miRNAs have been reported to be a part of HDACi-induced autophagy as well,- - this highlights the complex nature of miRNA-autophagy nexus in tumor formation as well as cancer therapy. The upregulation of miR-129-3p by nuclear factor erythroid two like-2 (Nrf-2) and the consequent repression of mTOR and activation of autophagy can contribute to chemoresistance in HDACi treatment. Thus, inhibition of Nrf2/miR-129-3p can suppress autophagy and promote chemosensitivity in HDACi-treated tumors. A comprehensive microarray-based study carried out in human pancreatic cancer cells (PANC-1) has identified miR-663a-5p and miR-154-5p as being downregulated when autophagy is suppressed by chloroquine diphosphate. Besides establishing 
these two miRNAs essential for autophagy in PANC-1 cells, this microarray analysis has identified long non-coding RNAs (lncRNAs), circular RNAs (circRNAs), and multiple regulated genes by these miRNAs, thus linking autophagy to other cellular pathways in pancreatic cancer models. This study further validates the deep involvement of miRNAs in the regulation of autophagy in pancreatic cancer [85]. This dichotomy in the effect of miRNA/autophagy regulation on cancer therapy, accompanied by the involvement of lncRNAs, has been reported in liver cancers as well. The levels of lncRNA metastasis-associated lung adenocarcinoma transcript 1 (MALAT1) are significantly higher in hepatocellular carcinoma (HCC) tumors than in neighboring tissues. Silencing of MALAT1 in HCC cell lines (Huh7, HepG2, LO2) restores apoptosis and autophagy and the levels of miR-146a. The latter (miR-146a), suppressed by MALAT1, is essential as a tumor suppressor in this context-miR146a silencing reverses the anti-tumor effects of MALAT1 inhibition. Since miR-146a blocks PI3K/AKT/mTOR to induce autophagy and apoptosis, MALAT1 and miR-146a together represent a viable target for therapies against HCC [58]. Similarly, miR-423-5p plays a vital role in the induction of autophagy in sorafenib-treated hepatocellular carcinoma (HCC) cell lines, and miR-638 promotes apoptosis and autophagy in liver cancer cells (SNU-182, 387,423,449) by the post-transcriptional downregulation of enhancer of zeste homolog 2 (EZH-2) [71, 96]. Yet, in another study, miR-375 blocks ATG7 activity and mitophagy in hypoxic human HCC (Huh7, Hep3B) cell lines. This blunted autophagy response contributes to increased susceptibility of HCC cell lines and xenograft models to hypoxic stress. In a similar trend, the same miR-375 blocks ATG14-mediated autophagy in sorafenib-treated Huh7 and HepG2 cells and increase sensitivity to sorafenib $[8,91]$. In another study, overexpression of miR-132 in HCC cells and tissues blocks PIK3R3-dependent autophagy but promotes apoptosis. In this case, a lncRNA LINC00160 acts as a negative regulator of miR-132 and is expressed in significantly high levels in HCC samples and cells [98]. Thus, the complex cycle of different classes of non-coding RNAs plays a crucial role in the progression of tumors and determines the nature of therapies that can stall cell proliferation [29].

In gastric cancer (GC) tissues, long non-coding RNA (lncRNA) MALAT1 promotes autophagy and proliferation by downregulating the tumor suppressor miR-204. Overexpression of miR-204 in GC cell lines CTC105 and CTC141 downregulates transient receptor potential melastatin 3 (TRPM3), an activator of oncogenic autophagymiR-204 can thus be utilized as a target in GC therapy [68]. Another notable anti-autophagy tumor suppressor, miR-30a, increases sensitivity to imatinib (IM) in gastrointestinal stromal tumor (GIST) cells and mouse xenograft models. Beclin-1 is the specific target of miR-30a, and the levels of
Beclin-1 driven autophagy are higher in IM-resistant cells like GIST-882 than the sensitive GIST-1 cell line [14]. In addition, anti-autophagy miRNAs serve as tumor suppressors in other variants of digestive tumors, e.g., colorectal cancers (CRCs). A commonly used carcinostatic drug to treat CRCs, catalpol, elevates miR-34a levels in CRC tissues and cell lines (HCT116, HT29, SW620, SW480). As a result, miR-34a blocks Sirtuin-1 (SIRT1) expression, leading to impaired autophagy in catalpol-treated cells. Furthermore, this step is essential in inducing apoptosis in catalpol-treated CRC cell lines and (azoxymethane-induced) rat models [60]. However, miR-126 has an opposite effect on autophagy in CRC biopsy samples and cell lines (HCT116, SW260, SW480). miR-126 blocks mTOR, thus inducing autophagy and apoptosis, facilitating the inhibition of cell proliferation in tumors. Studies with Bafilomycin-1 establish that the anti-tumor apoptosis, in this case, is dependent on miR-126 mediated autophagy. In this context, it is interesting to note that in the two studies shown above, autophagy acts upstream of apoptosis, thus highlighting the importance of miRNAs in regulating the mutual crosstalk between cell death pathways in tumor resistance [86]. Similar crosstalk has also been observed in prostate cancer $(\mathrm{PCa})$ cells, where miR-381 blocks the reelin (RLEN)/PI3K/AKT/mTOR pathway and promotes autophagy and apoptosis. Since the endogenous levels of miR-381 are low in PCa cells, mimics can promote cell death, as has been done in this study on nude mice models [42].

In laryngeal carcinoma, miR-26b is activated, which leads to uninhibited cell proliferation. Downregulation of miR-26b in Hep-2 cells activates both autophagy and Caspase-3/9 dependent apoptosis, thus preventing cell proliferation. Furthermore, simultaneous downregulation of miR-26b and ULK-2 results in an even greater degree of autophagy and apoptosis in Hep-2 cells, thus highlighting the importance of miR-26b in the progression of laryngeal cancer [79].

In non-small cell lung cancer (NSCLC), miR-153-3p contributes to Gefitinib-sensitivity by suppressing ATG5 and hence the entire autophagy pathway. In human NSCLC cell lines PC-9 and HCC-827, low miR-153-3p and high autophagy ensure resistance against Gefitinib, which has been successfully reversed by activation of miR-153-3p [97].

In multiple myeloma cell lines (U266), autophagy and apoptosis exhibit an antagonistic relationship. The levels of miR-196-5p are higher, and that of miR-99a-5p are lower in U266 cells compared to normal plasma cells. As a result, elevated autophagy and low apoptosis are observed in U266 cells, which has been replicated in normal plasma cells by upregulation of miR-196-5p and inhibition of miR-99a-5p [66].

In osteosarcoma (OS) cell lines (MG63), resistance to cisplatin (CDDP) is a result of autophagy which ironically is upregulated by CDDP treatment. miR-22 reverses 
this effect by upregulating the PI3K/AKT/mTOR pathway and weakening the autophagy response in CDDP-treated normal MG63 cells and resistant MG63 (MG63/CDDP) cells. An interesting finding to emerge from this study is the comparable efficacy of miR-22 and autophagy inhibitor wortmannin in dampening the CDDP resistance in MG63 and MG63/CDDP cells. This finding underscores the importance of miRNA analysis in a different light—such investigations can also identify potential pharmacological molecules (wortmannin) that can aid in cancer therapy [52].

In glioblastoma cell lines, induction of autophagy by blocking the activity of the ATG7-specific miR-17 increases the sensitivity of these cells to anti-tumor drugs and radiotherapy. In T98G, inhibition of miR-17 upregulates autophagy and increases the sensitivity of these cells to Temozolomide (TMZ), a standard glioblastoma therapy. Furthermore, in U-373-MG, mi-17 downregulation makes the cells susceptible to low-dose ionizing radiation. Thus, miR-17 modulation could be helpful as potential targets for glioblastoma [16].

Although regulation of autophagy by miRNAs has been widely documented mostly in DKD and PKD, it is also observed in kidney cancers [28]. miR-100, a positive regulator of autophagy, is downregulated in renal cell carcinoma (RCC), the most common cancer of the kidneys. A low expression of miR-100 has been recorded in parallel to significant upregulation of anti-autophagy factors like nicotinamide adenine dinucleotide phosphate oxidase 4 (NOX4), mammalian target of rapamycin (mTOR), and downregulation of LC3 in tumors from RCC patients. In RCC cell lines like ACHN and 786-0, NOX4 activation has been effectively reversed by overexpression of miR-100, and the subsequent activation of autophagy blocked migration and proliferation in these cell lines [46] Another study that focuses on the most common RCC type, the clear cell RCC, has presented a different pattern. In ccRCC cell lines Caki-1, 2, and KJ29, high autophagy levels are simultaneously observed with a high level of the pro-autophagy miR501-5p. miR501-5p drives AMPK-dependent autophagy, leading to TP53 degradation, thus removing a checkpoint on uncontrolled cell proliferation. Inhibition of autophagy in this model restores the regular activity of TP53, leading to reduced cell proliferation and migration [57]. Another study shows a link between the tumor suppressor von Hippel-Lindau (VHL) gene and miR-204 in clear cell Renal Cell Carcinoma (ccRCC) human samples. miR-204, a negative regulator of the elongation step (LC3 lipidation) of autophagy, is downregulated in high-grade RCC tumors, and overexpression of VHL can restore the regular miR-204 activity. Thus, the VHL/miR204 axis serves to check the autophagy levels, and abrogation of this pathway leads to continued cell proliferation in ccRCC [53]. Although few in numbers, these studies can prove helpful as forerunners of miRNA-dependent therapies for renal cancers.

\section{Cardiovascular disorders}

Regulation of autophagy by miRNAs has been implicated in several cardiovascular disorders ranging from atherosclerosis, sleeping disorders, cardiomyopathy, myocardial infarction, cardiac fibrosis, cardiac hypertrophy, and heart failure [72]. Cardiomyopathy is a side effect often observed in patients receiving Doxorubicin, a widely used antitumor drug, and a primary reason cited has been the upregulation of autophagy. Overexpression of angiotensin-converting enzyme 2 (ACE2) has been shown to mitigate the effects of Doxorubicin, primarily through the attenuation of autophagy. Studies in primary rat ventricular cardiomyocytes and HC92 cells have demonstrated that the activity of anti-autophagy miR-30e is a vital component of the ACE-2 induced amelioration of cardiomyopathy in Doxorubicin treatment [35]. Sleeping disorders like obstructive sleep apnea/hypopnea syndromes (OSAHS) are characterized by cardiac endothelial dysfunction, and a significant trigger behind this phenotype is chronic intermittent hypoxia (CIH). miRNA CHIP analysis in a mouse CIH model of aortic endothelial injury demonstrates substantial upregulation of four and downregulation of two mi-RNAs. All these miRNAs are part of the pathways that regulate autophagy and apoptosis-inhibition of one such upregulated miR-193 blocks apoptosis and autophagy in the CIH model [45]. The downregulation of miR-145 in in vivo and in vitro models of acute myocardial infarction (AMI) led to increased apoptosis and suppressed autophagy. Inhibition of the specific target of miR-145-Akt3- and suppression of the lysosomal activity neutralizes miR-145 upregulation in in vitro models of AMI - thus miR-145 is a strong candidate for treating AMI [89].

The significance of miRNAs in autophagy has been deeply studied in atherosclerosis [72]. A Chinese herbal medicine Paeonol, extracted from Cortex Moutan roots, blocks autophagy in an in vivo model of atherosclerosis. Oxidized low-density lipoprotein (ox-LDL) induced vascular endothelial injury (VEC) in Sprague Dawley rats have been linked to Beclin-1 upregulation and induction of autophagy. The therapeutic effect of Paeonol on ox-LDL-treated animals relies on the suppression of autophagy, and a critical component of this response is miR-30a, a specific inhibitor of Beclin-1 [39]. Another study that focused on the mechanism of foam cell formation in human macrophage cells (THP-1) demonstrates that the upregulation of miR-761 following ox-LDL-treatment is detrimental to lipid accumulation. The effect on autophagy, in this case, is opposite to that of the Paeonol study described above. miR-761 
binds explicitly to mTOR and induces autophagy which also facilitates the downregulation of pro-inflammatory cytokines IL- $1 \beta$ and IL-18. Treatment of ox-LDL-treated THP- 1 cells with miR-761 mimic and an autophagy inhibitor (Chloroquine) shows that the anti-inflammatory effect of miR-761 is heavily dependent upon autophagy flux [75]. Finally, a non-canonical mode of post-translational modification by miR-126-5p in human umbilical vein vascular endothelial (HUVEC) cells has broadened the spectrum of the effect of miRNAs in atherosclerosis. In HUVECs exposed to high shear stress, a high level of autophagy ensures the formation of a complex between argonaute-2 (Ago-2), miR-126-5p, and Mex3a. This event promotes the nuclear localization of miR-126-5p, where it binds to Caspase- 3 in an aptamer-like fashion and blocks its dimerization. The anti-apoptotic effect of miR-126-5p is a crucial feature of reduced endothelial injury and can be harnessed as an essential target in atherosclerosis treatment [65].

\section{Diabetes and digestive and kidney diseases}

The role of miRNAs in the regulation of autophagy has been well documented in kidney injuries, polycystic kidney diseases as well as renal cancers. In ischemic kidney injury models of 6-8-week-old C57BL/6 males, increased autophagy is accompanied by a significant decrease in the levels of miR-20a-5p, an inhibitor of ATG16L1. The same pattern is observed in proximal tubular cells (HK-2) that had been exposed to hypoxic conditions. On further investigation, the hypoxia-inducible factor HIF- $\alpha$ emerges as an upstream negative regulator of MiR-20a-5p, thus identifying a tripartite HIF- $\alpha /$ miR-20a-5p/ATG16L1 axis which is important in the pathology of ischemic kidney injury [77]. In acute kidney injury (AKI) models, the production of hazardous molecules like advanced oxidation protein products (AOPPs) manifests as a reduction of protective autophagy in renal tubular epithelial cells (RTECs) like HK-2. An important application of stem cell therapy in renal injury has been to differentiate mesodermal stem cells into healthy tubular epithelial cells. Among the candidates forwarded by stem cell therapy, umbilical cord human mesenchymal stem cells (hUC-MSCs) have proven effective in restoring autophagy in AOPP-treated RETCs. This event is attributed to an upregulation of miR-145 and the subsequent induction of autophagy by the downregulation of the PI3K/AKT/mTOR pathway in AOPP-treated HK-2 cells co-cultured with hUCMSCs. This finding can be beneficial for stem cell therapy in renal injury models and trigger more studies investigating the role of miRNAs in this approach [87].

miRNAs also positively regulate autophagy in other EndStage Renal Diseases (ESRDs) like diabetic nephropathy. Low levels of a differentially expressed miR-18a-5p were recorded in 8-week-old diabetic $(d b / d b)$ mice compared to normal $(d b /+)$ mice. Glomerular injury is rescued by resveratrol (RSV) treatment in the renal cortex of diabetic $(\mathrm{db} /$ $d b$ ) mice and cultured human podocytes. Reversal of the diabetic phenotype, as mediated by RSV treatment, has been attributed to increased autophagy and a diminished rate of apoptosis. RSV treatment also restores miR-18a-5p levels in diabetic kidneys, and this has a profound effect on the activation of autophagy which has a protective effect on the kidneys in diabetic mice. Further exploration has revealed that miR-18a-5p blocks apoptosis and induced autophagy by exerting a transcriptional blockade on Ataxia telangiectasia mutated (ATM). Thus, the ATM/ miR-18a-5p/autophagy pathways can be harnessed as a potential target for the treatment of diabetic nephropathy [88]. In another model of diabetic glomerular hypertrophy, miR-192 has been identified as an essential factor in downregulating autophagy in the glomeruli of mouse models of both types I and II diabetes and primary mouse mesangial (MMC) cell cultures. Inhibition of miR-192 by the administration of a locked nucleic acid (LNA) anti-miR-192 successfully restored the expression levels of essential autophagy genes in the renal cortex of diabetic mice, and further exploration using a miR-192 KO diabetic mouse model has identified phosphorylated FoxO3a (p-FoxO3a) as a potential downstream target of miR-192. Finally, investigation in MMC cultures has also identified TGF-beta as an upstream regulator of miR-192 and miR-217, both of which are negative regulators of autophagy. This study has unearthed multiple therapeutic targets, notably the TGF-B/ miR-192/ pFoxO3a pathway, to treat diabetic glomerular hypertrophy [19]. The inhibitory effect of autophagoMIRs has also been reported to facilitate renal interstitial fibrosis (RIF), an essential hallmark of chronic kidney disease (CKD). An inhibitor of the essential autophagy gene $\operatorname{Atg} 5$, miR-376b, is responsible for the CKD phenotype in 6-8-week-old FVB/N male mice. Furthermore, it has been documented that suppression of macrophage autophagy is a crucial component of this phenotype. Investigations using miR-376b mimic and inhibitor have shown that deregulation of this miRNA rescues the CKD phenotype, primarily by removing the transcriptional block imposed on Atg5 and inducing apoptosis in kidney fibroblasts (KFB), thus abrogating KFB proliferation. Administration of Atg5 shRNA, with and without miR-376b inhibitor, also validates this finding. This study, which also shows increased ROS in mice treated with miR-376b inhibitor, highlights the importance of miRNAs in the crosstalk between different pathways of programmed cell death (autophagy, apoptosis) in the pathogenesis of the end-stage renal disease (ESRD) [90]. Renal tubulointerstitial fibrosis is also an essential feature of diabetic nephropathy (DN), a pathological condition already discussed above in the context of microRNAs regulating autophagy. As in CKD mouse models, another 
miRNA-miR-22-blocking the transcription of phosphatase and tensin homolog (PTEN)-mediated autophagy has been linked to renal fibrosis in rat models of DN. In renal tissues obtained from streptozotocin (STZ)-treated ten weeks old Sprague Dawley rats and rat kidney cells (NRK-52E) treated with high-glucose (HG), a high level of miR-22 coupled with low levels of autophagy has been demonstrated. miR-22 blocks PTEN-mediated autophagy and increases the levels of fibrosis markers collagen IV (Col IV) and $\alpha$-smooth muscle actin ( $\alpha$-SMA) in these models, thus acting as a bridge between fibrosis and autophagy. Treatment of HG-exposed NRK-52E cells with an inducer of autophagyrapamycin-reverses the high expression of fibrotic markers (Col IV), thus emphasizing the protective role of autophagy in DN-related fibrosis [100]. The involvement of miRNA pathways blocking autophagy in DN tissues and cell cultures has also been helpful for possible therapies. For instance, Oleanolic Acid (OA) - a projected remedy against $\mathrm{DN}-$ induces autophagy by suppressing miR-142-5p. Much like the miR-22 described above, miR-142-5p also blocks PTEN expression and thus reduces the levels of autophagy in DN mice kidneys and NRK-52E (HG-treated) cultures. The treatment of HG-exposed NRK-52E cells with 10uM OA (48 h) successfully reverses the increased miR-22 activity and restores PTEN activity and autophagy levels. OA treatment also suppresses the expression of renal fibrosis markers (Col IV, $\alpha$-SMA) in HG-treated NRK-52E cells, besides stopping the PI3K/AKT/mTOR pathway from repressing autophagy. This finding further underlines the importance of miRNA/PTEN/autophagy cascade in the progression of renal fibrosis in diabetic nephropathy [11]. In a similar study, Ursolic Acid (UA) reverses diabetic mesangial cell injury by downregulating miR-21, an inhibitor of phosphatase and tensin homolog (PTEN). In rat glomerular mesangial cells incubated in a high-glucose (HG) medium, i.e., a type I diabetes mimic, UA reduces miR-21 levels and expression of $\mathrm{PI} 3 \mathrm{~K} / \mathrm{AKT} / \mathrm{mTOR}$ pathway while upregulating PTEN and autophagy. This mechanism is also crucial to the suppression of renal fibrosis, and this finding is essential for evaluating miRNAs as potential therapies for DN/DKD [47, 50].

Recent investigations have also identified upstream regulators of miRNAs regulating autophagy in diabetic kidney disease (DKD). Reduced rate of autophagic degradation in two mice models (Akita and STZ-treated C57BL/6) have been linked to increased p53 and miR-214, which results in a decreased ULK1 phosphorylation, thus blocking the very first stage in autophagy. Studies with knockout models suggest that attenuation of the p53/miR-22 pathway in the proximal tubules (PT) effectively decreases renal fibrosis and hypertrophy and improves renal function. Findings from in vitro models (rat and human PT cell lines) also validate the involvement of the p53/mir-22/ULK1 pathway in the progression of DKD. Finally, data from renal biopsies have also confirmed the relevance of this pathway in DKD patients [51].

Polycystic kidney disease (PKD) represents another primary class of ESRD, making up about $10 \%$ of all cases. A member of the miR-106b 25 cluster-miR-25-3p-has been shown to downregulate autophagy (ATG14) in renal tissues from PKD (Pkdlfl/+; Ksp-Cre) mouse models. Inhibition of the miR-25-3p activity restores autophagy and blocks proliferation in renal tubular epithelial cells (isolated from PKD mice). This study is a testimonial to the immense potential of miRNAs regulating autophagy as possible targets for ESRD therapy [44].

Besides chronic diseases, the effect of miRNAs on the regulation of autophagy (and related pathways) in the kidney has been explored in environmental toxicity. Cadmium $(\mathrm{Cd})$ toxicity, a significant source of metal pollution in poultry animals, induces downregulation of miR-30a - an inhibitor of the endoplasmic reticulum chaperone GRP78-in kidney cells isolated from Cd-treated Hy-Line Brown laying hens. As a result, high endoplasmic reticulum stress (ERS), unfolded protein response (UPR), and autophagy is observed in chicken kidney cells. This critical study that links ERS/ UPR to autophagy as a downstream pathway of miR-30a also establishes Selenium (Se) as an antidote to Cd-toxicity. Selenium (Se) can neutralize the low miR-30a and the high ERS/autophagy levels in Cd-treated kidney cells. This study is a shining example of the importance of miRNAs in environmental disorders in non-mammalian kidneys. We can harness this knowledge to better livestock living conditions [69].

A significant correlation between miRNAs, known to block autophagy, and excessive fibrosis has been reported in chronic liver diseases like liver cirrhosis, echoing a similar pattern in chronic kidney diseases. Investigation in human samples from liver cirrhosis patients and carbon tetrachloride (CCL4) treated C57BL/6 (6-8 w) mouse models, low autophagy flux, and high miR-125a activity has been observed. In both cases, vitamin D receptor (VDR) - a known inducer of autophagy and a target of miR-125a-is downregulated, thus acting as a bridge between miRNA and autophagy. Neutralization of miR-125a activity by antagomirs restores VDR activity and autophagy flux and reduces the levels of fibrosis in CCL4-induced mouse hepatocytes. Hence, an in-depth investigation of the relation between miRNAs and autophagy can aid in treating chronic liver diseases [26].

\section{Infectious diseases}

Autophagy is an essential homeostatic process that plays a vital role in regulating innate immune responses during bacterial infection [70]. In infectious diseases, miRNAs play 
a crucial role in the modulation of autophagy and innate immune response in diverse ways, suggesting that miRNAs can be explored as targets for therapeutic approaches. In Mycobacterium tuberculosis (Mtb)-infected macrophages and monocytes, non-coding RNAs like miR-30a, miR125a-3p [32], miR-17-5p [34], miR-155 [27], miR-31 [27], miR-889 [10], miR-27a, and miR-144 [31] block different pathways of autophagy, thereby facilitating infection [30]. Since autophagy is generally regarded as a vital component of the antiviral innate immune response, miRNAs blocking autophagy are frequently upregulated in Mtb infection. In studies investigating $M$. bovis infections, miR-199a, 20a, 144-3p, 3619-5p, 15a, 142-5p, 21-3p, 30b-5p, 22-3p, 23a have been reported to block autophagosome maturation in macrophages. Besides, miRNAs regulating autophagy are overexpressed in Listeria (miR-146a, miR-125a, miR$155)$ and Helicobacter (mirR-30b, miR-30d, miR-155) infections [70]. Interestingly, high levels of miR-30c (inhibits ATG5) and miR-130a (inhibits ATG16L1) in exosomes released by intestinal epithelial cells (T84), infected with a clinical isolate (LF82) of Crohn's disease-associated adherent invasive $E$. coli (AIEC), reduced autophagy levels in recipient IEC cells. This data reveals an intriguing mechanism by which bacterial infection can induce miRNAs transfer between different cells via exosomes [37].

The regulation of autophagy by miRNAs has also been characterized in viral infections. Rotavirus infection triggers upregulation of a pro-autophagy miR-99b and downregulation of an anti-autophagy let-7 $\mathrm{g}$, and this upsurge in autophagy aids viral replication in vitro. miR-99b blocks mTOR to induce autophagy while the downregulation of let-7 g activates TSC1, activating proviral autophagy through diverse pathways. miR-99b inhibitor and let-7 g mimic block viral replication in vitro, presenting a unique case study where two non-coding RNAs are regulated differently in viral infection [54]. miR-155 expression is reduced in hepatoblastoma cells (HepG2215) steadily infected with Hepatitis B (HBV) compared to uninfected HepG2 cells. However, overexpression of miR-155 increases viral replication and antibody production in HepG2215, pointing towards a possible feedback mechanism where endogenous miR-155 might be downregulated in infected cells as part of an antiviral mechanism. Overexpression of miR-155 modulates the suppressor of cytokine signaling (SOCS1)/ $\mathrm{Akt} / \mathrm{mTOR}$ pathway and induced autophagy. In this case, autophagy aids in replicating the virus, and SOCS1 is in a feedback relation with Interferon-G (INF-G). It is perhaps no surprise that miR-155 also exhibits a feedback response to HBV infection. In liver biopsy samples, miR-155 expression increases with elevated levels of fibrosis in chronic hepatitis $\mathrm{C}$ (CHC) but shows no alteration in the different stages of autoimmune hepatitis (AIH). The same study reports that an investigation of miRNA analysis in $24 \mathrm{CHC}$ and $18 \mathrm{AIH}$ samples demonstrate no significant difference in the levels of miR-155, -204, -224; however, miR-101 is elevated in CHC compared to AIH. These studies show that the miRNAs modulating autophagy exert a complicated effect on viral infections, and further investigation is required $[13,74]$.

\section{Inflammation and immune system}

Interferon- $\beta$ induces miR- 1 to prevent the aggregation of huntingtin protein in mouse primary cortical neuronal cells. In this case, miR-1 induces autophagy by the downregulation of the RAB GTPase activating protein TBC1D15 (Tbc7 in C. elegans). Induction of autophagy by suppressing TBC1D15, a negative regulator of the pro-autophagy RAB7, prevents polyglutamine aggregation [56]. A more recent study on in vivo and in vitro models of Parkinson's Disease (PD) shows the upregulation of miR-3473b and inflammatory cytokines (TNF- $\alpha, \mathrm{IL}-1 \beta$ ), which results in the inhibition of autophagy. miR-3473b, in this case, blocks autophagy (ULK-1) by the specific inhibition of the triggering receptor expressed on myeloid cells (TREM) 2. Since TREM2 has been reported to block the activation of microglia, a significant hallmark of PD, miR-3473b favors the inflammatory pathogenesis of PD. These findings present miRNAs as an essential target in diseases caused by the accumulation of toxic proteins (Huntington's, Parkinson's, Alzheimer's) [49]. In other studies, miRNAs are reported to regulate the expression of cytokines. In in vivo and in vitro models of thyroiditis, miR-125a induces the expression of pro-inflammatory cytokines (TNF- $\alpha$, IL- $1 \beta$, IL-6, IL-8) and downregulates autophagy (PI3K/mTOR pathway). Besides, miR-125a also induces apoptosis and cell proliferation in these models [9]. The negative correlation between autophagy-related fibrosis and miR-30a is an essential driving factor in the progression of asthma in patients, mouse models, and lung epithelial cells. In these models, miR-30a, while blocking ATG5 to suppress autophagy, also negates the fibrotic effect of IL-33 in lung epithelial cells, thus linking autophagy to inflammatory fibrosis [38]. A more direct convergence of inflammation and autophagy as downstream pathways of miRNAs have been noted in human monocytic leukemia cells (THP-1) exposed to fine particulate matters (PM2.5), a leading environmental hazard responsible for pulmonary inflammation conditions like asthma and COPD. miR-146a-5p downregulates both autophagy genes (ATG-5, ULK1) and inflammatory cytokines (IL-8) in PM2.5-treated THP-1 cells. Besides, miR-146a-5p also blocks activators of autophagy-related inflammation like interleukin-1 associated kinase-1 (IRAK1) and tumor necrosis factor receptorassociated factor 6 (TRAF6). The effect of miRNAs on both autophagy and inflammation makes them a tenable target for therapy of inflammation-related disorders [67]. 


\section{Osteoarthritis}

In light of the findings presented above, where miRNAs regulate autophagy in aging-related processes and inflammation, it is interesting to note that miRNA regulation of autophagy has been widely reported in osteoarthritis (OA) $[17,94]$. Treatment of primary chondrocytes (obtained from Sprague-Dawley rats) with tumor necrosis factor-a (TNF-a) is a commonly used OA model characterized by low cell viability and a high level of apoptosis. miR-4262 activity is elevated in this model and is a primary driver of the autophagy suppression observed in this OA model. Sirtuin-1 (SIRT1) being a specific target of miR-4262, autophagy is downregulated following the activation of the $\mathrm{PI} 3 \mathrm{~K} / \mathrm{mTOR}$ pathway [73]. Similarly, miR-375 specifically inhibits ATG2B in chondrocytes collected from OA patients and OA mouse models. The upregulation of miR-375 in OA chondrocytes is concomitant with increased apoptosis, endoplasmic reticulum stress (ERS), and the administration of miR375 antagomirs induces autophagy in these models [40]. Inhibition or downregulation of miR-4262 and miR-375 could thus be utilized as a potential OA therapy. In other studies, miRNAs facilitating autophagy have been reported to be downregulated in OA models. In human chondrocytes, miR140-5p and miR-149 target fucosyltransferase 1 (FUT-1) and induces autophagy to neutralize the effect of interleukin $1 \mathrm{~b}$ (IL-1B) induced apoptosis in human chondrocytes [82, 83]. miR-335-5p expression is significantly diminished in human OA chondrocytes, and overexpression using miR-335-5p downregulates the inflammatory mediators like IL-1B, IL-8, and TNF- $\alpha$. Furthermore, miR-335-5p activates antiinflammatory autophagy (BECN1, ATG5, ATG7), which is crucial for OA therapy [102]. In a knee osteoarthritis (KOA) rat model, circulating let-7e is significantly diminished in the peripheral serum, and let-7e expression is reduced in chondrocytes. Suppression of let-7e is concomitant with increased apoptosis and reduced autophagy. According to these results, miRNAs activating autophagy can be utilized for OA treatment [23].

\section{Neurological disorders}

The involvement of miRNAs in the regulation of autophagy holds the key to the treatment of neurological disorders. In rat primary hippocampal microglial cells, hemoglobin promotes miR-144 mediated suppression of MTOR and the activation of autophagy [84]. Furthermore, miR-144 mediated downregulation of mTOR facilitates pro-inflammatory response and promotes brain injury in ICH mouse models. Thus, the miR-144/mTOR pathway can be utilized for intracerebral hemorrhage (ICH) therapy [93]. Although the mTOR-dependent pathway of autophagy has been chiefly identified as the target of miRNAs, other pathways are also regulated in aging-related neurological disorders. A MAPKdependent way of miRNA-regulated autophagy has been determined in Alzheimer's Disease (AD) patients and mouse models, thus presenting a non-mTOR model that can be used for therapies. The suppression of miR-101a in AD models facilitates MAPK1-dependent autophagy, which can be instrumental in neurodegeneration [41]. As with other disorders, miRNAs regulating autophagy can also be beneficial for $\mathrm{AD}$ treatment, as demonstrated in resveratrol-mediated treatment [33].

\section{Discussion}

This review summarizes recent findings on the effect of miRNAs on autophagy in aging-related conditions, different cancers, cardiovascular complications, diabetes and kidney diseases, infectious diseases, immune disorders, osteoarthritis, and neurodegeneration (Table1). The spectrum of this review includes antagonistic miRNAs that degrade and thereby downregulate autophagy genes and proautophagy miRNAs that suppress negative down regulators of autophagy like the PI3K-AKT-mTOR pathway (Fig. 1). Hence, there is an immense prospect of utilizing these miRNAs as therapeutic targets. Furthermore, in some of these reports, the miRNAs do not solely regulate autophagy but also the crosstalk between autophagy and other cell death pathways (apoptosis) and stress (ER stress) pathways. This finding further expands the scope of miRNA-based targeted therapy beyond the realms of autophagy in these models. Finally, it is encouraging that certain traditional medicines and small molecule drugs are reported to employ the miRNA-autophagy network in some of the disease models. It validates the efficacy of miRNAs as feasible targets in disease prevention. 
Table 1 A list of miRNAs regulating autophagy in different pathophysiological conditions

\begin{tabular}{|c|c|c|c|}
\hline miRNA & Target & Pathological Condition & References \\
\hline miR-83 & CUP5 & Ageing (in C. elegans) & {$[103]$} \\
\hline miR-21 & PTEN & Invertebral disc degeneration (IDD) & {$[43,80]$} \\
\hline miR-129-5p & BECN-1 & Invertebral disc degeneration (IDD) & {$[101]$} \\
\hline miR-185 & GALECTIN-3 & Invertebral disc degeneration (IDD) & [95] \\
\hline miR-153-3p & ATG-5, 7 & IDD & {$[81]$} \\
\hline miR-202-5p & ATG-7 & IDD & [12] \\
\hline miR-210 & ATG-7 & IDD & {$[76]$} \\
\hline miR-144 & mTOR & Intracerebral hemorrhage (ICH) & {$[93]$} \\
\hline miR-101a & MAPK1 & Alzheimer's Disease (AD) & [41] \\
\hline $\operatorname{miR}-4262$ & SIRT1 & Osteoarthritis (OA) & {$[73]$} \\
\hline $\operatorname{miR}-375$ & ATG2B & Osteoarthritis (OA) & {$[40]$} \\
\hline $\begin{array}{l}\operatorname{miR}-140-5 p \\
\text { miR-149 }\end{array}$ & FUT-1 & Osteoarthritis (OA) & {$[82,83]$} \\
\hline $\operatorname{miR}-335-5 p$ & IL-1B, IL-8, TNF- $\alpha$ & Osteoarthritis (OA) & {$[102]$} \\
\hline miR-1 & TBC1D15 & Huntington's Disease & {$[56]$} \\
\hline $\operatorname{miR}-3473 b$ & ULK-1/ TREM2 & Parkinson's Disease (PD) & [49] \\
\hline miR-125a & mTOR-dependent autophagy & Thyroiditis & [9] \\
\hline miR-30a & ATG5 & Asthma & [38] \\
\hline miR-146a-5p & ATG-5, ULK1, IL-8, IRAK-1, TRAF-6 & Asthma, COPD & {$[67]$} \\
\hline miR-145 & Akt3 & Acute myocardial infarction (AMI) & [89] \\
\hline miR-30a & BECN-1 & Atherosclerosis & [39] \\
\hline miR-761 & mTOR & Atherosclerosis & [75] \\
\hline miR-126-5p & Caspase-3 & Atherosclerosis & {$[65]$} \\
\hline miR-30a & BECN-1 & $\begin{array}{l}\text { Human breast cancer cell lines (MDA-MB-468); Lung cancer } \\
\text { cell lines (T98G); Glioma cell lines (H1299) }\end{array}$ & [104] \\
\hline miR-638 & DACT3 & $\begin{array}{l}\text { Esophageal squamous cancer (KYSE450) and breast cancer } \\
\text { (MCF-7) cell lines }\end{array}$ & {$[62]$} \\
\hline miR-224-5p & SMAD4 & Breast cancer cells (MCF-7, MDA-MB-231) & [15] \\
\hline miR-23b & ATG12 & Pancreatic cancer cells (PC-1) & [78] \\
\hline $\operatorname{miR} 221$ & HDAC & Pancreatic cancer cells (PC-1) & [92] \\
\hline miR-129-3p & mTOR & Chemoresistance in HDACi treated PC-1 & {$[85]$} \\
\hline miR-146a & PI3K/AKT/mTOR & Hepatocellular Carcinoma cell lines (Huh7, HepG2, LO2) & {$[58]$} \\
\hline miR-423-5p, miR-638 & $\mathrm{EZH}-2$ & $\begin{array}{l}\text { Hepatocellular carcinoma (HCC) cell lines, Liver cancer cells } \\
\text { (SNU-182, 387,423,449) }\end{array}$ & {$[71,96]$} \\
\hline miR-375 & ATG7, ATG14 & Human HCC cell lines (Huh7, Hep3B, HepG2) & [91] \\
\hline miR-204 & TRPM3 & Gastric Cancer (GC) cells (CTC105, CTC141) & [68] \\
\hline miR-30a & BECN-1 & $\begin{array}{l}\text { Gastrointestinal stromal tumor (GIST) cells, mouse xenograft } \\
\text { models }\end{array}$ & {$[14]$} \\
\hline $\operatorname{miR}-34 a$ & SIRT-1 & $\begin{array}{l}\text { Colorectal Cancer (CRC) tissues and cell lines (HCT116, } \\
\text { HT29, SW620, SW480) }\end{array}$ & {$[60]$} \\
\hline miR-126 & mTOR & $\begin{array}{l}\text { Colorectal Cancer (CRC) biopsy samples and cell lines } \\
\text { (HCT116, SW260, SW480) }\end{array}$ & {$[86]$} \\
\hline miR-381 & Reelin (RLEN)/PI3K/AKT/mTOR pathway & Prostate Cancer $(\mathrm{PCa})$ cells & {$[42]$} \\
\hline miR-153-3p & ATG5 & $\begin{array}{l}\text { Non-small cell lung cancer (NSCLC) cell lines (PC-9; HCC- } \\
\text { 827) }\end{array}$ & [97] \\
\hline miR-22 & PI3K/AKT/mTOR-dependent autophagy & $\begin{array}{l}\text { Cisplatin (CDDP) treated osteosarcoma (OS) cell lines } \\
\text { (MG63) }\end{array}$ & {$[52]$} \\
\hline miR-17 & ATG7 & Glioblastoma cell lines (T98G, U-373-MG) & {$[16]$} \\
\hline $\operatorname{miR} 501-5 p$ & TP53 & $\begin{array}{l}\text { Clear cell renal cell carcinoma (ccRCC) cell lines (Caki-1, 2, } \\
\text { and } \mathrm{KJ} 29 \text { ) }\end{array}$ & {$[57]$} \\
\hline miR-204 & Elongation (LC3 lipidation) of autophagy & ccRCC human samples & {$[3,53]$} \\
\hline
\end{tabular}


Table 1 (continued)

\begin{tabular}{|c|c|c|c|}
\hline miRNA & Target & Pathological Condition & References \\
\hline miR-20a-5p & ATG16L1 & $\begin{array}{l}\text { Ischemic kidney injury models (C57BL/6), Hypoxic proximal } \\
\text { tubular cells (HK-2) }\end{array}$ & {$[77]$} \\
\hline $\operatorname{miR}-145$ & $\mathrm{PI} 3 \mathrm{~K} / \mathrm{AKT} / \mathrm{mTOR}$ & Acute kidney injury (AKI) models (HK-2) & [87] \\
\hline miR-18a-5p & ATM & $\begin{array}{l}\text { Diabetic Glomerular Nephropathy (human samples and mice } \\
\text { models) }\end{array}$ & [88] \\
\hline miR-192 & Phosphorylated FoxO3a (p-FoxO3a) & Diabetic Glomerular Nephropathy (mice models) & [19] \\
\hline miR-376b & ATG5 & $\begin{array}{l}\text { Renal Interstitial Fibrosis (RIF) associated with mice models } \\
\text { of chronic kidney disease (CKD) }\end{array}$ & {$[90]$} \\
\hline miR-22 & PTEN-mediated autophagy & $\begin{array}{l}\text { Renal tubulointerstitial Fibrosis (RIF) associated with rat } \\
\text { models of diabetic nephropathy (DN) }\end{array}$ & {$[100]$} \\
\hline miR-142-5p & PTEN-mediated autophagy & DN mice kidneys and in vitro models & $(11)$ \\
\hline $\operatorname{miR}-21$ & PTEN-mediated autophagy & DN/ diabetic kidney disease (DKD) & {$[47,50]$} \\
\hline miR-214, miR-22 & ULK1 & DKD—human biopsy samples, rat models, cells & {$[51]$} \\
\hline $\operatorname{miR}-25-3 p$ & ATG14 & Polycystic Kidney Disease (PKD) mouse models & {$[44]$} \\
\hline miR-30a & GRP78 (endoplasmic reticulum chaperone) & In vitro models of Cadmium $(\mathrm{Cd})$ toxicity in kidneys & [69] \\
\hline $\operatorname{miR}-125 \mathrm{a}$ & VDR (vitamin D receptor) & $\begin{array}{l}\text { Chronic Liver Disease (Liver Cirrhosis) — human samples, } \\
\text { and mouse models }\end{array}$ & {$[26]$} \\
\hline $\operatorname{miR}-30 \mathrm{c}$ & ATG5 & $\begin{array}{l}\text { Crohn's disease-associated adherent invasive } E \text {. coli (AIEC) } \\
\text { infected intestinal epithelial cells }\end{array}$ & {$[37]$} \\
\hline miR-130a & ATG16L1 & $\begin{array}{l}\text { Crohn's disease-associated adherent invasive E. coli (AIEC) } \\
\text { infected intestinal epithelial cells }\end{array}$ & {$[37]$} \\
\hline $\operatorname{miR}-99 b$ & mTOR & Rotavirus infection & [54] \\
\hline miR-155 & $\begin{array}{l}\text { Suppressor of cytokine signaling (SOCS1)/ } \\
\text { Akt/mTOR pathway }\end{array}$ & Hepatitis B (HBV) infection & [13] \\
\hline
\end{tabular}

Fig. 1 The most commonly functioning miRNAs regulating the transcriptional expression of upstream activators (indicated by green dotted box) and inhibitors (red dotted box) of autophagy

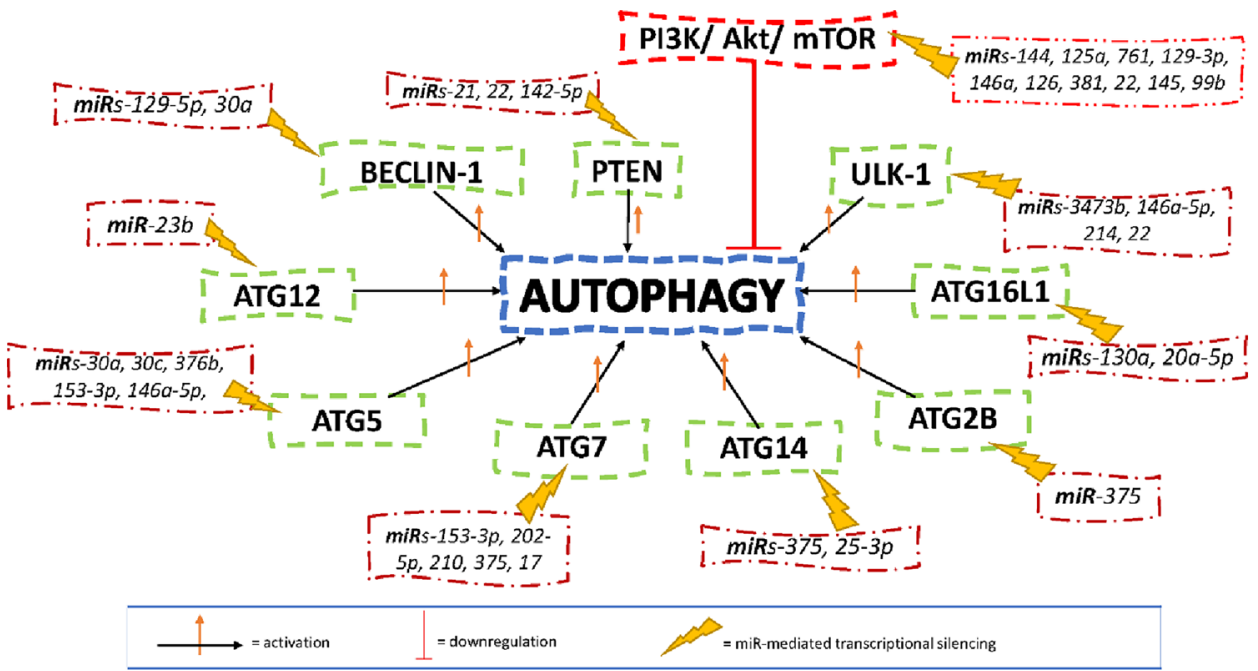

Acknowledgements I sincerely thank the editorial team, especially associate editor Dr. Somnath Paul, for extending me the opportunity to write this review. I am also grateful to Yale School of Medicine (Internal Medicine - Nephrology) for the library and internet facilities. Last but not the least, I am deeply indebted to my Ph.D. mentor Dr. Zahra Zakeri (City University of New York) for initiating me in the field of autophagy and cellular homeostasis.

\section{Declarations}

Conflict of interest Not Applicable.

Ethical approval Not Applicable.

Consent to participate Not Applicable.

Consent for publication Not Applicable. 


\section{References}

1. Abdellatif M, Ljubojevic-Holzer S, Madeo F, Sedej S. Autophagy in cardiovascular health and disease. Prog Mol Biol Transl Sci. 2020;172:87-106.

2. Akkoc Y, Gozuacik D. MicroRNAs as major regulators of the autophagy pathway. Biochim Biophys Acta Mol Cell Res. 2020;1867:118662.

3. Atala A. Re: VHL-Regulated miR-204 suppresses tumor growth through inhibition of LC3B-mediated autophagy in renal clear cell carcinoma. J Urol. 2012;188:2434.

4. Balzano F, Campesi I, Cruciani S, Garroni G, Bellu E, et al. Epigenetics, stem cells, and autophagy: exploring a path involving miRNA. Int J Mol Sci. 2019;20:5091.

5. Bartel DP. MicroRNAs: genomics, biogenesis, mechanism, and function. Cell. 2004;116:281-97.

6. Bhatia D, Choi ME. Autophagy in kidney disease: advances and therapeutic potential. Prog Mol Biol Transl Sci. 2020;172:107-33.

7. Buque Martinez A, Galluzzi L. Preface-Autophagy: An old mechanism with new challenges ahead. Prog Mol Biol Transl Sci. 2020;172:xv-xix.

8. Chang Y, Yan W, He X, Zhang L, Li C, et al. miR-375 inhibits autophagy and reduces viability of hepatocellular carcinoma cells under hypoxic conditions. Gastroenterology. 2012;143:177-87.

9. Chen D, Huang X, Lu S, Deng H, Gan H, et al. miRNA-125a modulates autophagy of thyroiditis through PI3K/Akt/mTOR signaling pathway. Exp Ther Med. 2019;17:2465-72.

10. Chen DY, Chen YM, Lin CF, Lo CM, Liu HJ, Liao TL. MicroRNA-889 inhibits autophagy to maintain mycobacterial survival in patients with latent tuberculosis infection by targeting TWEAK. mBio. 2020;11:e03045.

11. Chen J, Cui Y, Zhang N, Yao X, Wang Z, Yang L. Oleanolic acid attenuated diabetic mesangial cell injury by activation of autophagy via miRNA-142-5p/PTEN signaling. Cytotechnology. 2019;71:925-33.

12. Chen J, Sun Q, Liu GZ, Zhang F, Liu CY, et al. Effect of miR202-5p-mediated ATG7 on autophagy and apoptosis of degenerative nucleus pulposus cells. Eur Rev Med Pharmacol Sci. 2020;24:517-25.

13. Chen L, Ming X, Li W, Bi M, Yan B, et al. The microRNA-155 mediates hepatitis B virus replication by reinforcing SOCS1 signalling-induced autophagy. Cell Biochem Funct. 2020;38:436-42.

14. Chen W, Li Z, Liu H, Jiang S, Wang G, et al. MicroRNA-30a targets BECLIN-1 to inactivate autophagy and sensitizes gastrointestinal stromal tumor cells to imatinib. Cell Death Dis. 2020;11:198

15. Cheng Y, Li Z, Xie J, Wang P, Zhu J, et al. MiRNA-224-5p inhibits autophagy in breast cancer cells via targeting Smad4. Biochem Biophys Res Commun. 2018;506:793-8.

16. Comincini S, Allavena G, Palumbo S, Morini M, Durando F, et al. microRNA-17 regulates the expression of ATG7 and modulates the autophagy process, improving the sensitivity to temozolomide and low-dose ionizing radiation treatments in human glioblastoma cells. Cancer Biol Ther. 2013;14:574-86.

17. D’Adamo S, Cetrullo S, Minguzzi M, Silvestri Y, Borzi RM, Flamigni F. MicroRNAs and autophagy: fine players in the control of chondrocyte homeostatic activities in osteoarthritis. Oxid Med Cell Longev. 2017;2017:3720128.

18. Deretic V, Klionsky DJ. Autophagy and inflammation: a special review issue. Autophagy. 2018;14:179-80.

19. Deshpande S, Abdollahi M, Wang M, Lanting L, Kato M, Natarajan R. Reduced autophagy by a microRNA-mediated signaling cascade in diabetes-induced renal glomerular hypertrophy. Sci Rep. 2018;8:6954.

20. Dinesh Kumar N, Smit JM, Reggiori F. Strategies employed by viruses to manipulate autophagy. Prog Mol Biol Transl Sci. 2020;172:203-37.

21. Engedal N, Zerovnik E, Rudov A, Galli F, Olivieri F, et al. From oxidative stress damage to pathways, networks, and autophagy via MicroRNAs. Oxid Med Cell Longev. 2018;2018:4968321.

22. Esteller M. Non-coding RNAs in human disease. Nat Rev Genet. 2011;12:861-74.

23. Feng L, Feng C, Wang CX, Xu DY, Chen JJ, et al. Circulating microRNA let7e is decreased in knee osteoarthritis, accompanied by elevated apoptosis and reduced autophagy. Int J Mol Med. 2020;45:1464-76.

24. Filipowicz W, Bhattacharyya SN, Sonenberg N. Mechanisms of post-transcriptional regulation by microRNAs: are the answers in sight? Nat Rev Genet. 2008;9:102-14.

25. Frankel LB, Lund AH. MicroRNA regulation of autophagy. Carcinogenesis. 2012;33:2018-25.

26. He W, Ni W, Zhao L, Wang X, Liu L, Fan Z. MicroRNA-125a/ VDR axis impaired autophagic flux and contributed to fibrosis in a CCL4-induced mouse model and patients with liver cirrhosis. Life Sci. 2021;264:118666.

27. Holla S, Kurowska-Stolarska M, Bayry J, Balaji KN. Selective inhibition of IFNG-induced autophagy by Mir155- and Mir31-responsive WNT5A and SHH signaling. Autophagy. 2014;10:311-30.

28. Huang K, Sun X, Wu H, Zhao J, Jian Y, et al. The regulating effect of autophagy-related MiRNAs in kidney, bladder, and prostate cancer. J Oncol. 2021;2021:5510318.

29. Jiang PC, Bu SR. Clinical value of circular RNAs and autophagyrelated miRNAs in the diagnosis and treatment of pancreatic cancer. Hepatobiliary Pancreat Dis Int. 2019;18:511-6.

30. Kim JK, Kim TS, Basu J, Jo EK. MicroRNA in innate immunity and autophagy during mycobacterial infection. Cell Microbiol. 2017;19:e12687.

31. Kim JK, Lee HM, Park KS, Shin DM, Kim TS, et al. MIR144* inhibits antimicrobial responses against Mycobacterium tuberculosis in human monocytes and macrophages by targeting the autophagy protein DRAM2. Autophagy. 2017;13:423-41.

32. Kim JK, Yuk JM, Kim SY, Kim TS, Jin HS, et al. MicroRNA125a inhibits autophagy activation and antimicrobial responses during mycobacterial infection. J Immunol. 2015;194:5355-65.

33. Kou X, Chen N. Resveratrol as a natural autophagy regulator for prevention and treatment of Alzheimer's disease. Nutrients. 2017;9:927.

34. Kumar R, Sahu SK, Kumar M, Jana K, Gupta P, et al. MicroRNA 17-5p regulates autophagy in Mycobacterium tuberculosis-infected macrophages by targeting Mcl-1 and STAT3. Cell Microbiol. 2016;18:679-91.

35. Lai L, Chen J, Wang N, Zhu G, Duan X, Ling F. MiRNA30e mediated cardioprotection of ACE2 in rats with Doxorubicin-induced heart failure through inhibiting cardiomyocytes autophagy. Life Sci. 2017;169:69-75.

36. Lan T, Shiyu H, Shen Z, Yan B, Chen J. New insights into the interplay between miRNAs and autophagy in the aging of intervertebral discs. Ageing Res Rev. 2021;65:101227.

37. Larabi A, Dalmasso G, Delmas J, Barnich N, Nguyen HTT. Exosomes transfer miRNAs from cell-to-cell to inhibit autophagy during infection with Crohn's disease-associated adherent-invasive E. coli. Gut Microbes. 2020;11:1677-94.

38. Li BB, Chen YL, Pang F. MicroRNA-30a targets ATG5 and attenuates airway fibrosis in asthma by suppressing autophagy. Inflammation. 2020;43:44-53. 
39. Li C, Yang L, Wu H, Dai M. Paeonol inhibits oxidized lowdensity lipoprotein-induced vascular endothelial cells autophagy by upregulating the expression of miRNA-30a. Front Pharmacol. 2018;9:95.

40. Li H, Li Z, Pi Y, Chen Y, Mei L, et al. MicroRNA-375 exacerbates knee osteoarthritis through repressing chondrocyte autophagy by targeting ATG2B. Aging (Albany NY). 2020;12:7248-61.

41. Li Q, Wang Y, Peng W, Jia Y, Tang J, et al. MicroRNA-101a regulates autophagy phenomenon via the MAPK pathway to modulate Alzheimer's-associated pathogenesis. Cell Transplant. 2019;28:1076-84.

42. Liao W, Zhang Y. MicroRNA-381 facilitates autophagy and apoptosis in prostate cancer cells via inhibiting the RELNmediated PI3K/AKT/mTOR signaling pathway. Life Sci. 2020;254:117672.

43. Lin H, Zhang W, Zhou T, Li W, Chen Z, et al. Mechanism of microRNA-21 regulating IL-6 inflammatory response and cell autophagy in intervertebral disc degeneration. Exp Ther Med. 2017;14:1441-4.

44. Liu G, Kang X, Guo P, Shang Y, Du R, et al. miR-25-3p promotes proliferation and inhibits autophagy of renal cells in polycystic kidney mice by regulating ATG14-Beclin 1. Ren Fail. 2020;42:333-42.

45. Liu KX, Chen GP, Lin PL, Huang JC, Lin X, et al. Detection and analysis of apoptosis- and autophagy-related miRNAs of mouse vascular endothelial cells in chronic intermittent hypoxia model. Life Sci. 2018;193:194-9.

46. Liu X, Zhong L, Li P, Zhao P. 2020. MicroRNA-100 Enhances autophagy and suppresses migration and invasion of renal cell carcinoma cells via disruption of NOX4-dependent mTOR Pathway. Clin Transl Sci.

47. Lu X, Fan Q, Xu L, Li L, Yue Y, et al. Ursolic acid attenuates diabetic mesangial cell injury through the up-regulation of autophagy via miRNA-21/PTEN/Akt/mTOR suppression. PLoS One. 2015;10:e0117400.

48. Luo P, Gao F, Niu D, Sun X, Song Q, et al. The role of autophagy in chondrocyte metabolism and osteoarthritis: a comprehensive research review. Biomed Res Int. 2019;2019:5171602.

49. Lv Q, Zhong Z, Hu B, Yan S, Yan Y, et al. MicroRNA-3473b regulates the expression of TREM2/ULK1 and inhibits autophagy in inflammatory pathogenesis of Parkinson disease. J Neurochem. 2021;157:599-610.

50. Ma J, Wang Y, Xu HT, Ren N, Zhao N, et al. MicroRNA: a novel biomarker and therapeutic target to combat autophagy in diabetic nephropathy. Eur Rev Med Pharmacol Sci. 2019;23:6257-63

51. Ma Z, Li L, Livingston MJ, Zhang D, Mi Q, et al. p53/microRNA-214/ULK1 axis impairs renal tubular autophagy in diabetic kidney disease. J Clin Invest. 2020;130:5011-26.

52. Meng CY, Zhao ZQ, Bai R, Zhao W, Wang YX, et al. MicroRNA22 mediates the cisplatin resistance of osteosarcoma cells by inhibiting autophagy via the PI3K/Akt/mTOR pathway. Oncol Rep. 2020;43:1169-86.

53. Mikhaylova O, Stratton Y, Hall D, Kellner E, Ehmer B, et al. VHL-regulated MiR-204 suppresses tumor growth through inhibition of LC3B-mediated autophagy in renal clear cell carcinoma. Cancer Cell. 2012;21:532-46.

54. Mukhopadhyay U, Chanda S, Patra U, Mukherjee A, Rana S, et al. Synchronized orchestration of miR-99b and let-7g positively regulates rotavirus infection by modulating autophagy. Sci Rep. 2019;9:1318.

55. Munz C. Autophagy in immunity. Prog Mol Biol Transl Sci. 2020;172:67-85.
56. Nehammer C, Ejlerskov P, Gopal S, Handley A, Ng L, et al. Interferon-beta-induced miR-1 alleviates toxic protein accumulation by controlling autophagy. Elife. 2019;8:e49930.

57. Patergnani S, Guzzo S, Mangolini A, dell'Atti L, Pinton P, Aguiari G. The induction of AMPK-dependent autophagy leads to P53 degradation and affects cell growth and migration in kidney cancer cells. Exp Cell Res. 2020;395:112190.

58. Peng N, He J, Li J, Huang H, Huang W, et al. Long noncoding RNA MALAT1 inhibits the apoptosis and autophagy of hepatocellular carcinoma cell by targeting the microRNA-146a/PI3K/ Akt/mTOR axis. Cancer Cell Int. 2020;20:165.

59. Pulakat L, Chen HH. Pro-senescence and anti-senescence mechanisms of cardiovascular aging: cardiac MicroRNA regulation of longevity drug-induced autophagy. Front Pharmacol. 2020;11:774.

60. Qiao PF, Yao L, Zeng ZL. Catalpolmediated microRNA34a suppresses autophagy and malignancy by regulating SIRT1 in colorectal cancer. Oncol Rep. 2020;43:1053-66.

61. Racanelli AC, Choi AMK, Choi ME. Autophagy in chronic lung disease. Prog Mol Biol Transl Sci. 2020;172:135-56.

62. Ren Y, Chen Y, Liang X, Lu Y, Pan W, Yang M. MiRNA-638 promotes autophagy and malignant phenotypes of cancer cells via directly suppressing DACT3. Cancer Lett. 2017;390:126-36.

63. Rodriguez-Muela N. Autophagy in motor neuron diseases. Prog Mol Biol Transl Sci. 2020;172:157-202.

64. Saha S, Panigrahi DP, Patil S, Bhutia SK. Autophagy in health and disease: a comprehensive review. Biomed Pharmacother. 2018;104:485-95.

65. Santovito D, Egea V, Bidzhekov K, Natarelli L, Mourao A, et al. Noncanonical inhibition of caspase- 3 by a nuclear microRNA confers endothelial protection by autophagy in atherosclerosis. Sci Transl Med. 2020;12:eaaz294.

66. Shang J, Chen ZZ, Wang ZH, Wei TN, Wu WB, Chen WM. Association of miRNA-196b-5p and miRNA-99a-5p with autophagy and apoptosis in multiple myeloma cells. Zhonghua Xue Ye Xue Za Zhi. 2018;39:766-72.

67. Shang Y, Liu Q, Wang L, Qiu X, Chen Y, An J. microRNA146a-5p negatively modulates PM2.5 caused inflammation in THP-1 cells via autophagy process. Environ Pollut. 2021;268:115961

68. Shao G, Zhao Z, Zhao W, Hu G, Zhang L, et al. Long noncoding RNA MALAT1 activates autophagy and promotes cell proliferation by downregulating microRNA-204 expression in gastric cancer. Oncol Lett. 2020;19:805-12.

69. Shi Q, Jin X, Fan R, Xing M, Guo J, et al. Cadmium-mediated miR-30a-GRP78 leads to JNK-dependent autophagy in chicken kidney. Chemosphere. 2019;215:710-5.

70. Silwal P, Kim YS, Basu J, Jo EK. The roles of microRNAs in regulation of autophagy during bacterial infection. Semin Cell Dev Biol. 2020;101:51-8.

71. Stiuso P, Potenza N, Lombardi A, Ferrandino I, Monaco A, et al. MicroRNA-423-5p promotes autophagy in cancer cells and is increased in serum from hepatocarcinoma patients treated with sorafenib. Mol Ther Nucleic Acids. 2015;4:e233.

72. Sun T, Li MY, Li PF, Cao JM. MicroRNAs in cardiac autophagy: small molecules and big role. Cells. 2018;7:104

73. Sun W, Li Y, Wei S. miR-4262 regulates chondrocyte viability, apoptosis, autophagy by targeting SIRT1 and activating PI3K/ AKT/mTOR signaling pathway in rats with osteoarthritis. Exp Ther Med. 2018;15:1119-28.

74. Szekerczes T, Gogl A, Illyes I, Mandl J, Borka K, et al. Autophagy, mitophagy and microrna expression in chronic hepatitis $\mathrm{C}$ and autoimmune hepatitis. Pathol Oncol Res. 2020;26:2143-51.

75. Wang C, Yang W, Liang X, Song W, Lin J, et al. MicroRNA-761 modulates foam cell formation and inflammation 
through autophagy in the progression of atherosclerosis. Mol Cell Biochem. 2020;474:135-46.

76. Wang C, Zhang ZZ, Yang W, Ouyang ZH, Xue JB, et al. MiR210 facilitates ECM degradation by suppressing autophagy via silencing of ATG7 in human degenerated NP cells. Biomed Pharmacother. 2017;93:470-9.

77. Wang IK, Sun KT, Tsai TH, Chen CW, Chang SS, et al. MiR-20a-5p mediates hypoxia-induced autophagy by targeting ATG16L1 in ischemic kidney injury. Life Sci. 2015;136:133-41.

78. Wang P, Zhang J, Zhang L, Zhu Z, Fan J, et al. MicroRNA 23b regulates autophagy associated with radioresistance of pancreatic cancer cells. Gastroenterology. 2013;145:1133-43.

79. Wang S, Guo D, Li C. Downregulation of miRNA-26b inhibits cancer proliferation of laryngeal carcinoma through autophagy by targeting ULK2 and inactivation of the PTEN/AKT pathway. Oncol Rep. 2017;38:1679-87.

80. Wang WJ, Yang W, Ouyang ZH, Xue JB, Li XL, et al. MiR-21 promotes ECM degradation through inhibiting autophagy via the PTEN/akt/mTOR signaling pathway in human degenerated NP cells. Biomed Pharmacother. 2018;99:725-34.

81. Wang XB, Wang H, Long HQ, Li DY, Zheng X. LINC00641 regulates autophagy and intervertebral disc degeneration by acting as a competitive endogenous RNA of miR-153-3p under nutrition deprivation stress. J Cell Physiol. 2019;234:7115-27.

82. Wang Z, Hu J, Pan Y, Shan Y, Jiang L, et al. miR-140-5p/ miR-149 affects chondrocyte proliferation, apoptosis, and autophagy by targeting FUT1 in osteoarthritis. Inflammation. 2018;41:959-71.

83. Wang Z, Hu J, Pan Y, Shan Y, Jiang L, et al. Correction to: miR140-5p/miR-149 affects chondrocyte proliferation, apoptosis, and autophagy by targeting FUT1 in osteoarthritis. Inflammation. 2019;42:1515-6.

84. Wang Z, Yuan B, Fu F, Huang S, Yang Z. Hemoglobin enhances miRNA-144 expression and autophagic activation mediated inflammation of microglia via mTOR pathway. Sci Rep. 2017;7:11861.

85. Wei DM, Jiang MT, Lin P, Yang H, Dang YW, et al. Potential ceRNA networks involved in autophagy suppression of pancreatic cancer caused by chloroquine diphosphate: a study based on differentiallyexpressed circRNAs, IncRNAs, miRNAs and mRNAs. Int J Oncol. 2019;54:600-26.

86. Wei L, Chen Z, Cheng N, Li X, Chen J, et al. MicroRNA-126 inhibit viability of colorectal cancer cell by repressing mTOR induced apoptosis and autophagy. Onco Targets Ther. 2020;13:2459-68.

87. Xiang J, Jiang T, Zhang W, Xie W, Tang X, Zhang J. Human umbilical cord-derived mesenchymal stem cells enhanced HK-2 cell autophagy through MicroRNA-145 by inhibiting the PI3K/AKT/mTOR signaling pathway. Exp Cell Res. 2019;378:198-205.

88. Xu XH, Ding DF, Yong HJ, Dong CL, You N, et al. Resveratrol transcriptionally regulates miRNA-18a-5p expression ameliorating diabetic nephropathy via increasing autophagy. Eur Rev Med Pharmacol Sci. 2017;21:4952-65.

89. Yan L, Guo N, Cao Y, Zeng S, Wang J, et al. miRNA145 inhibits myocardial infarctioninduced apoptosis through autophagy via Akt3/mTOR signaling pathway in vitro and in vivo. Int J Mol Med. 2018;42:1537-47.
90. Yang S, Abdulla R, Lu C, Zhang L. Inhibition of microRNA$376 \mathrm{~b}$ protects against renal interstitial fibrosis via inducing macrophage autophagy by upregulating Atg5 in mice with chronic kidney disease. Kidney Blood Press Res. 2018;43:1749-64.

91. Yang S, Wang M, Yang L, Li Y, Ma Y, et al. MicroRNA-375 targets ATG14 to inhibit autophagy and sensitize hepatocellular carcinoma cells to sorafenib. Onco Targets Ther. 2020;13:3557-70.

92. Yang Y, Sun Y, Wang H, Li H, Zhang M, et al. MicroRNA-221 induces autophagy through suppressing HDAC6 expression and promoting apoptosis in pancreatic cancer. Oncol Lett. 2018;16:7295-301.

93. Yu A, Zhang T, Zhong W, Duan H, Wang S, et al. miRNA-144 induces microglial autophagy and inflammation following intracerebral hemorrhage. Immunol Lett. 2017;182:18-23.

94. Yu Y, Zhao J. Modulated autophagy by MicroRNAs in osteoarthritis chondrocytes. Biomed Res Int. 2019;2019:1484152.

95. Yun Z, Wang Y, Feng W, Zang J, Zhang D, Gao Y. Overexpression of microRNA-185 alleviates intervertebral disc degeneration through inactivation of the $\mathrm{Wnt} / \mathrm{beta}$-catenin signaling pathway and downregulation of Galectin-3. Mol Pain. 2020;16:1744806920902559.

96. Zhang H, Liang H, Wu S, Zhang Y, Yu Z. MicroRNA-638 induces apoptosis and autophagy in human liver cancer cells by targeting enhancer of zeste homolog 2 (EZH2). Environ Toxicol Pharmacol. 2021;82:103559.

97. Zhang W, Dong YZ, Du X, Peng XN, Shen QM. MiRNA-153-3p promotes gefitinib-sensitivity in non-small cell lung cancer by inhibiting ATG5 expression and autophagy. Eur Rev Med Pharmacol Sci. 2019;23:2444-52.

98. Zhang W, Liu Y, Fu Y, Han W, Xu H, et al. Long non-coding RNA LINC00160 functions as a decoy of microRNA-132 to mediate autophagy and drug resistance in hepatocellular carcinoma via inhibition of PIK3R3. Cancer Lett. 2020;478:22-33.

99. Zhang X, Yang K, Le W. Autophagy and motor neuron diseases. Adv Exp Med Biol. 2020;1207:53-74.

100. Zhang Y, Zhao S, Wu D, Liu X, Shi M, et al. MicroRNA-22 promotes renal tubulointerstitial fibrosis by targeting PTEN and suppressing autophagy in diabetic nephropathy. J Diabetes Res. 2018;2018:4728645.

101. Zhao K, Zhang Y, Kang L, Song Y, Wang K, et al. Methylation of microRNA-129-5P modulates nucleus pulposus cell autophagy by targeting Beclin-1 in intervertebral disc degeneration. Oncotarget. 2017;8:86264-76.

102. Zhong G, Long H, Ma S, Shunhan Y, Li J, Yao J. miRNA-335-5p relieves chondrocyte inflammation by activating autophagy in osteoarthritis. Life Sci. 2019;226:164-72.

103. Zhou Y, Wang X, Song M, He Z, Cui G, et al. A secreted microRNA disrupts autophagy in distinct tissues of Caenorhabditis elegans upon ageing. Nat Commun. 2019;10:4827.

104. Zhu H, Wu H, Liu X, Li B, Chen Y, et al. Regulation of autophagy by a beclin 1-targeted microRNA, miR-30a, in cancer cells. Autophagy. 2009;5:816-23.

Publisher's Note Springer Nature remains neutral with regard to jurisdictional claims in published maps and institutional affiliations. 